\title{
3D-Hit: fast structural comparison of proteins on multicore architectures
}

\author{
Ł. Bieniasz-Krzywiec • M. Cytowski • \\ L. Rychlewski • D. Plewczynski
}

Received: 30 August 2012 / Accepted: 3 October 2013 / Published online: 27 October 2013

(C) The Author(s) 2013. This article is published with open access at Springerlink.com

\begin{abstract}
D-Hit is a well established method for rapid detection of structural similarities between proteins, which is widely used in various bioinformatics web servers (MetaServer, GRDB, 3D-Fun, Rosetta, etc.). The algorithm decomposes proteins into set of overlaping segments of 9-13 residues, then tries to match them using root mean square distance metric. The best aligned pairs of segments are selected as seeds for futher analysis. Those initial hits are expanded by iterative process in order to construct the global structural alignment by concatenating pairs of matching segments. The method has the same accuracy as the other state-of-the-art structural comparison algorithms (LGscore2, DALI), yet it provides much faster processing times, and can be used in a high-throughput setup as the structural module of bioinformatics pipelines. The method is optimized in terms of speed and accuracy to work on novel computer architectures, such as PowerXCell8i and Sun Constellation System. Here, we provide the source code of the 3D-Hit program, describe selected architectures on which the software was ported, present programing models, point out significant porting steps and sumarize performance comparisons.
\end{abstract}

Keywords Proteins · IBM cell · Structure comparison · Bioinformatics · Optimization

\footnotetext{
Ł. Bieniasz-Krzywiec · M. Cytowski · D. Plewczynski ( $₫)$

Interdisciplinary Centre for Mathematical and Computational Modelling,

University of Warsaw (ICM), Pawińskiego 5a, 02-106 Warsaw, Poland

e-mail: darman@icm.edu.pl

L. Rychlewski

BioInfoBank Institute, Limanowskiego 24A16, 60-744 Poznan, Poland
} 


\section{Introduction}

The PowerXCell8i is a pioneering microprocessor architecture supposed to bridge the gap between conventional desktop computers and specialized high-performance machines. It has been designed to support very wide range of applications. The Cell processor consists of nine processor elements operating on a shared and coherent memory. Functions of those processors are specialized into two types, i.e., a PowerPC Processing Element (PPE), which usually acts as a controller and is designed to run operating system and eight Synergistic Processing Elements (SPEs), which handle computational workload and are optimized for SIMD code execution. PowerXCell8i processors are usually available as a dual processor nodes within the IBM QS22 blades. This gives a shared memory programming environment with 16 computational cores. Moreover due to its specific architecture, Cell processor achieves very good performance results with relatively low power consumption (in terms of performance per Watt). As a result, Nautilus [1]—cluster of IBM QS22 blades based on Cell processors installed at Interdisciplinary Centre for Mathematical and Computational Modelling (ICM) has been ranked as No. 1 in two editions of The Green500 List [2] of the world most energy-efficient supercomputers.

The Sun Constellation System, another high-performance computer available at ICM, is a cluster based on a x86 architecture. This powerful machine consists of blades packed into special-purpose racks, which are tied together with highly-efficient InfiniBand connections. Each blade is equipped with four AMD Quad-Core Opteron $835 \mathrm{X}$ processors (which gives 16 computational cores per blade) and up to $32 \mathrm{~Gb}$ of memory.

In this paper we describe two fast implementations of an efficient scanning method for detecting structural similarities between proteins. The first of them is an application designed for the PowerXCell8i processors. The second takes advantage of OpenMP and therefore can be executed on virtually all architectures providing shared memory access, including the Sun Constellation System. Thus, we compared two shared memory systems with different architectures but the same number of computational cores. The algorithm used in our programs was originally created by Plewczyński et al. $[3,4,11,12]$

The original code, destined to execute on x86 architecture, was ported using two different frameworks: Cell SDK with its SPE library (for PowerXCell8i architecture) and OpenMP (for parallel computers with shared memory). The Cell-accelerated application achieved an overall speedup of 12 over single threaded version executing on 1 SPE core. This level of performance was obtained with the use of all 16 SPU cores available within IBM QS22 blade. However, the program parallelized with OpenMP library performed even better in terms of the final walltime achieved during benchmarking tests. In the course of our work we encountered very interesting aspects of parallel programming and learned how to identify parts of code whose performance could benefit from novel high-performance architectures. 


\section{Structural alignment}

It has been discovered that the three dimensional structure of a protein is more conserved during the process of evolution than its primary sequence [5]. Therefore, the comparison of 3D structures of two proteins makes it possible to establish distant evolutionary relationships, even between very diverged proteins. As a result, the 3D structural alignment of proteins increases our understanding of more distant evolutionary relationships $[6,7]$. The correspondence between structural and functional classification enables scientists to determine functions of various newly discovered folds and whole protein families. Structural similarity can suggest evolutionary links between protein families, which can result in more detailed functional annotation of a given protein at molecular level. Moreover, the structural comparison can guide the experimental structure determination process, by tracing shifts in low resolution models. The aforementioned reasons make the structural alignment the very important part of bioinformatics every-day work.

On the other hand, the size of Protein Data Bank [8] is growing rapidly doubling every 18 months. This huge amount of structural data needs very fast and accurate computer programs to deal with the extraction of structural information and comparison of the new proteins with the previously annotated ones. Those programs should enable not only structure-to-structure search, but also alignment over all proteins from the whole database on a real time basis. So far, such computations has been performed by several state-of-the-art methods, including 3DHit code. Because of the overwhelming and constantly growing amount of processing to be performed, scientists requested the support of the Joint Cell Competence Centre [10]. Due to an ongoing collaboration between ICM and IBM, it was decided to port the 3DHit code, inter alia, to the PowerXCell8i architecture and use Cell based machines as a computational facility.

The purpose of this article is to present how the 3DHit program has been ported and tuned on novel architectures and how processing performance of accelerated versions compares to the $\mathrm{x} 86$ implementation.

\section{Overview of the algorithm}

3 DHit program provides the structural alignment of two proteins. It uses in-house customised version of the Smith-Waterman dynamic programming algorithm combined with intensive three-dimensional rotation and translation routines that align two geometrical objects in order to minimize the root mean square distance computed for all heavy atoms from both proteins. The flow chart of entire sequential program is presented in Fig. 1. The most time consuming part of the code is the preparation of structural alignment matrix which is carried out by the main loop of the program and is computed by examining local similarities between protein sequences. Alignment matrix is used at the end of the program, it serves as input data for the Smith-Waterman algorithm to compute the final global alignment of the whole proteins. To identify similarity substructures of two proteins, the program compares parts of their chains with a fixed length of 256 amino acids that we called segments. The 3 DHit program analyses 
Fig. 1 The flow chart of the scalar (starting) version of the 3D-Hit program

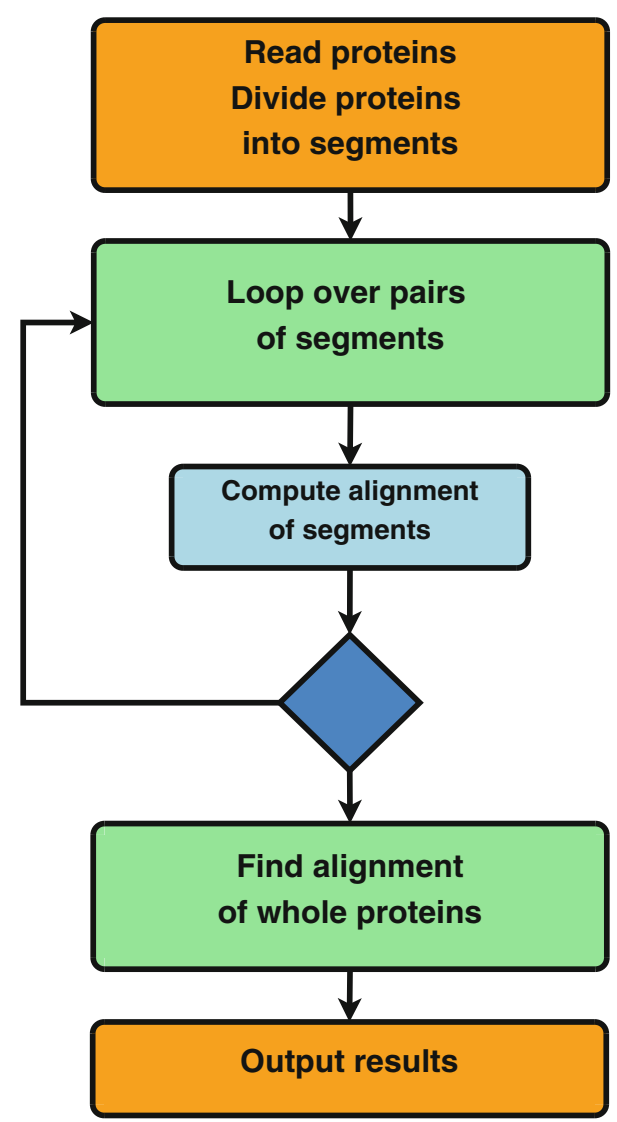

structural similarities between each pair of segments through two successive steps (see pseudo-code of Algorithm 1).

First, it decides whether central parts of segments, which we call seeds, are similar enough to proceed with further computations. Seeds are very short subsequences with 13 amino acids. The 3 DHit algorithm makes rotations and translations of alpha carbon atoms of both seeds in order to minimize the root mean square deviation (RMSD) between them. Second, if the structural similarity of the two seeds is high enough, the algorithm starts to analyze two longer continuous parts of the main chains centered on seeds. It uses a rotation matrix and a translation vector for a Cartesianspace superimposition of the two seeds to rotate and translate these large segments. Next, it defines the similarity matrix for the dynamic programming in the following way. If two alpha carbon atoms taken from the superimposed large segments are close enough in space (below 3A), it assigns 1 to their similarity score or 0 , otherwise. Then alignment score based on such similarity score matrix is computed. If the alignment score is large enough, algorithm passes this pair of segments on to the next filter. The whole procedure is repeated for subsequences of 100, 200 and 256 amino acids centered on seeds. 
For each pair of segments, the resulting score-number of superimposed alpha carbon atoms in the aligned segments - is recorded in an additional final alignment matrix. At the end, this final matrix is used to find the best alignment of whole proteins. If no pair of segments did pass the filter sequence just described, the overall score is set to 0 .

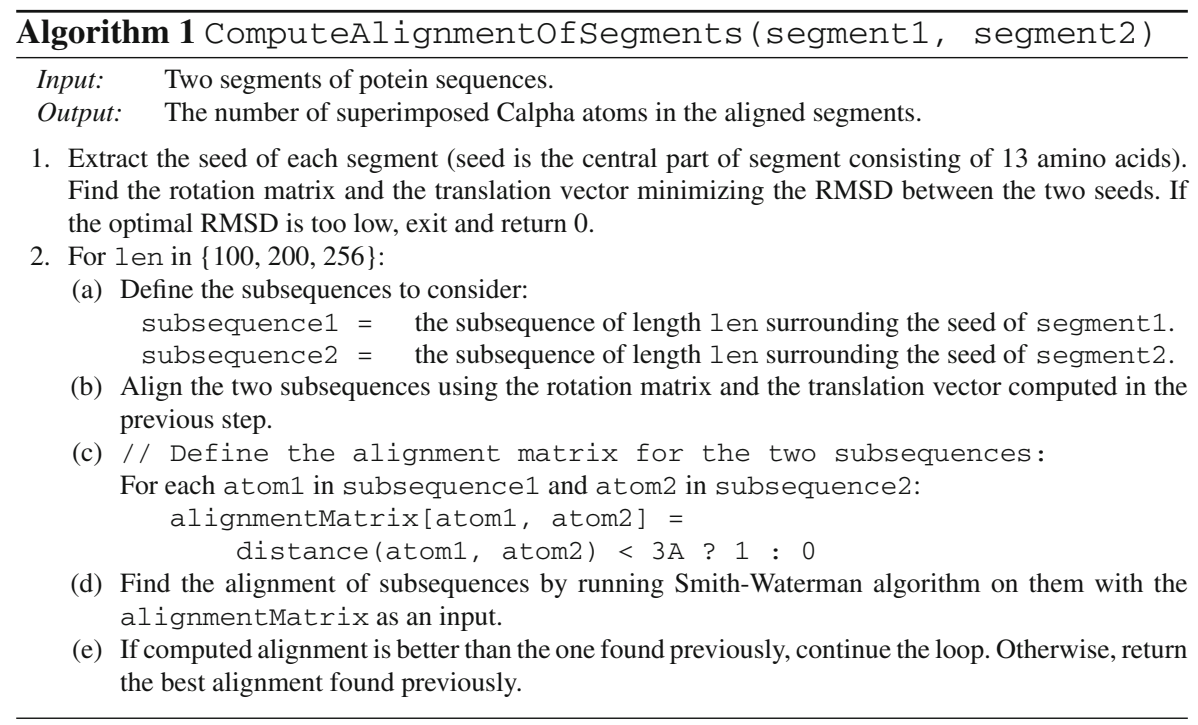

\section{Porting process}

After profiling the original version of the 3 DHit program we found out that the most time consuming part of the code was a function implementing algorithm, which compares two given segments. Intrinsically, the algorithm was executed in sequence with each pair of segments as a parameter. As a result, program spent more than $90 \%$ of its time in that function. We decided to accelerate that program section by a parallelization process based on the libspe 2 library model for Cell and the OpenMP model for x86 architectures (see Fig.2).

\subsection{Parallel scheme}

Computations for each pair of segments can be performed independently with no communication occurring between working threads. Nevertheless, at the end the main thread must collect all partial results for each pair of segments and compute the final result based on them. We wondered whether those extra computation steps performed on partial results would cause a new bottleneck. In the case of the Cell implementation, the final result computing is handled by the PPE. In the case of OpenMP version, the final result is processed by the master thread. Fortunately, it turned out that accelerated versions of 3 DHit program spends only a fraction of percent of their execution times computing final result. 


\subsection{PowerXCell8i implementation}

We used the Cell SDK and its SPE library to implement this simple parallel scheme. The PPE processor executes the main application thread. Its role is to read and preprocess the two protein sequence descriptions, create SPE contexts, run appropriate working threads, collect partial results and finally compute and return the final score and alignment. Each SPE processor gets its set of segment pairs. Then, it consecutively loads the descriptions of two segments into local memory space (Local Store) for each pair of segments from the set, executes function comparisons and stores the result back into main memory.

\subsubsection{Memory issue}

Porting most of the scientific applications to SPE processor is a rather challenging task because of the size limitation of Local Store, which is only $256 \mathrm{~KB}$. This small space must be wisely administrated, because it has to accommodate both program instructions and operating data. Moreover, the only way to load and store data to and from Local Store is a Direct Memory Access (DMA) mechanism. It gives programmer a great deal of control, but on the other hand it is not simple to use.

The 3 DHit code is unfortunately memory consuming. In the original version each execution of comparing algorithm requires memory for at least $256^{2}$ floating point numbers and $256^{2}$ short integer values. It is of course too much for the Local Store. That is why we had to perform some memory optimizations including compression and introduction of bit operations. The resulting code was slightly slower and less accurate than the original one, however thanks to those sacrifices, we were able to fit our program to Local Store.

\subsubsection{SIMD optimizations}

In its original version, 3 DHit program spends nearly $30 \%$ of its execution time calculating rotation matrices and translation vectors for Cartesian-space superimposition of pairs of segments. The part of code responsible for that task turned out to be a good candidate for a vectorization process due to the quantity of simple algebraic operations.

First of all, we decided to take advantage of the auto SIMDizing abilities of GCC compiler by switching on option -ftree-vectorize. At first, it did not help much since the code was too complex to allow the automatic analysis by the compiler. Therefore, we followed the guidelines proposed in [9] to simplify the code.

Even after using specific compiler directives, the compiler was still unable to automate loop vectorization. We decided to tune the remaining parts of code manually by instruction substitution. The main vector operations used in the SPE computational kernel were spu_add, spu_mul, spu_madd and spu_splats. All of these instructions were operating on float 4 entry vectors, so we could speed up the vectorized loops of the appropriate steps of the algorithm by a factor of about 4 . The result of this effort is presented in Table 1. 
Table 1 Performance results of SIMD and noSIMD versions

\begin{tabular}{llll}
\hline Version & Compiler & Average time & Speedup \\
\hline PowerXCell8i, noSIMD, 1 SPE & GCC (-03) & 1.546 & 1.0 \\
PowerXCell8i, noSIMD, 16 SPEs & GCC (-03) & $0.181 \mathrm{~s}$ & 8.541 \\
PowerXCell8i, SIMD, 16 SPEs & GCC (-03 -ftree-vectorize) & $0.164 \mathrm{~s}$ & 9.427 \\
\hline
\end{tabular}

Table 2 Performance results of various versions of work load management for the SPE threads

\begin{tabular}{lll}
\hline Version & Compiler & Average time \\
\hline Randomization on PPE, 16 SPEs & GCC (-03 -ftree-vectorize $)$ & $0.247 \mathrm{~s}$ \\
Dynamic distribution, 16 SPEs & GCC (-03 -ftree-vectorize $)$ & $0.370 \mathrm{~s}$ \\
Static permutation, 16 SPEs & GCC (-03 -ftree-vectorize $)$ & $0.164 \mathrm{~s}$ \\
\hline
\end{tabular}

\subsubsection{Efficient implementation of the parallel scheme}

In our first approach to implement chosen parallel scheme, we met very interesting problem. At the beginning we were assigning jobs for the computational kernels arbitrarily. Each SPE program was given a consecutive sequence of pairs of segments to operate on. Nevertheless, it was a wrong decision because of the inefficient load balancing. As described before, the algorithm for comparing segments does not always behave in the same manner. For example, the algorithm may finish very quickly if seeds have poor similarity rate. Moreover, cutoffs may occur also during the analysis of longer subsequences surrounding the seeds, as noted in the step 2 of the Algorithm 1. That is why it is important to assign each SPE with more or less the same amount of real work, which may not necessarily mean the same amount of pairs of segments.

We tried many different ways to fulfil this requirement. First of all, we decided to divide the whole set of tasks into 16 random subsets on the PPE side of application. Each SPE program operated on one of those randomly chosen work packages. This solution allowed the achievement of a very good load balancing, but on the other hand, to a drastic slowing down in the rate of PPE thread processing resulting in an unsustainable increase of time execution of about $15 \%$.

By contrast, the use of PPE thread as a management resource allows the workload to be distributed coherently according to computations needs. In this approach each idle SPE asks PPE for a new package for processing. We implemented and tested a few versions of such processing model by taking advantage of SPE mailboxes as well as advanced DMA transfers with double buffering. Unfortunately non of them met our expectations, because of the slowdown caused by the increase in communication load.

In the last approach, we have decided to choose an arbitrary random permutation and assign it statically to each SPE kernel as a constant. It allowed us the complete eradication of communication bottlenecks between PPE and SPE and to achieve reasonable load balancing. The performance comparison of all of these methods is presented in Table 2. 


\subsubsection{Other optimizations}

Each SPE program is executed once at the beginning and serves as a computational facility for many tasks. Thanks to that we are able to eliminate time needed for SPE context creation. In addition, we used the DMA double buffering mechanism. While SPE is carrying on computations, its Memory Controller can coherently load the next portions of data from the main memory. This simple idea allowed us to overlap main memory load and store operations with computations.

In comparison to the single SPE, we achieved an overall speedup of 6.14 while executing on 8 SPEs and 9.39 while executing on 16 SPEs. In addition, running two parallel instances of the 3DHit program, each using 8 SPEs, on the QS22 server equipped with two Cell chips allowed us to reach an average speedup of 12 .

\subsection{OpenMP implementation}

The implementation of a programming model based on OpenMP is very simple. The whole set of pairs of segments is dynamically (and automatically) distributed among OpenMP threads. Each such thread executes an algorithm similar to the one described in the PowerXCell8i's section. It gets a pair of segments, computes an answer and stores the result in the specified place in the main memory.

In spite of its simplicity, the OpenMP implementation of our application appeared to be very efficient and accurate. In comparison to the single-threaded version, we achieved an overall speedup of 4.37 while executing on 8 cores. Taking advantage of the same schema as the one used with PowerXCell8i processors, we ran two parallel instances of 3 DHit program, each using 8 threads, on blades equipped with 16 processor units, which gave an average speedup of 8.5 over the initial x 86 version.

\section{Performance results}

\subsection{PowerXCell8i code analysis}

We have used a spu_timing facility to analyze and tune the computational kernels of the 3DHit Cell implementation. Results presented in Tables 3 and 4 show that we achieved very good scaling over increasing number of working SPE threads. The part of code that can be parallelized represents about $97.994 \%$ of the execution time spent by the single-threaded version of 3 DHit. We achieve an overall speedup of almost 9 that is only a little below the theoretical maximum expected by the Amdahl's Law, i.e.

Table 3 Profile results - percentage of time spent in particular sections of the program

\begin{tabular}{lrrr}
\hline Part of code & 1 SPE (\%) & 8 SPEs (\%) & 16 SPEs (\%) \\
\hline Preparing data & 0.004782 & 0.142613 & 0.359532 \\
Creating SPE threads & 0.121318 & 7.627334 & 21.029137 \\
Waiting for threads & 97.844704 & 80.333420 & 62.397312 \\
Computing global result & 0.019680 & 0.139842 & 0.219315 \\
\hline
\end{tabular}


Table 4 Profile

results—absolute time spent in particular sections of the program

\begin{tabular}{llll}
\hline Part of code & 1 SPE $(\mathrm{s})$ & 8 SPEs $(\mathrm{s})$ & 16 SPEs $(\mathrm{s})$ \\
\hline Preparing data & 0.000051 & 0.000247 & 0.000466 \\
Creating SPE threads & 0.001170 & 0.012919 & 0.027042 \\
Waiting for threads & 1.492583 & 0.207126 & 0.114413 \\
Computing global result & 0.000527 & 0.000571 & 0.000619 \\
\hline
\end{tabular}
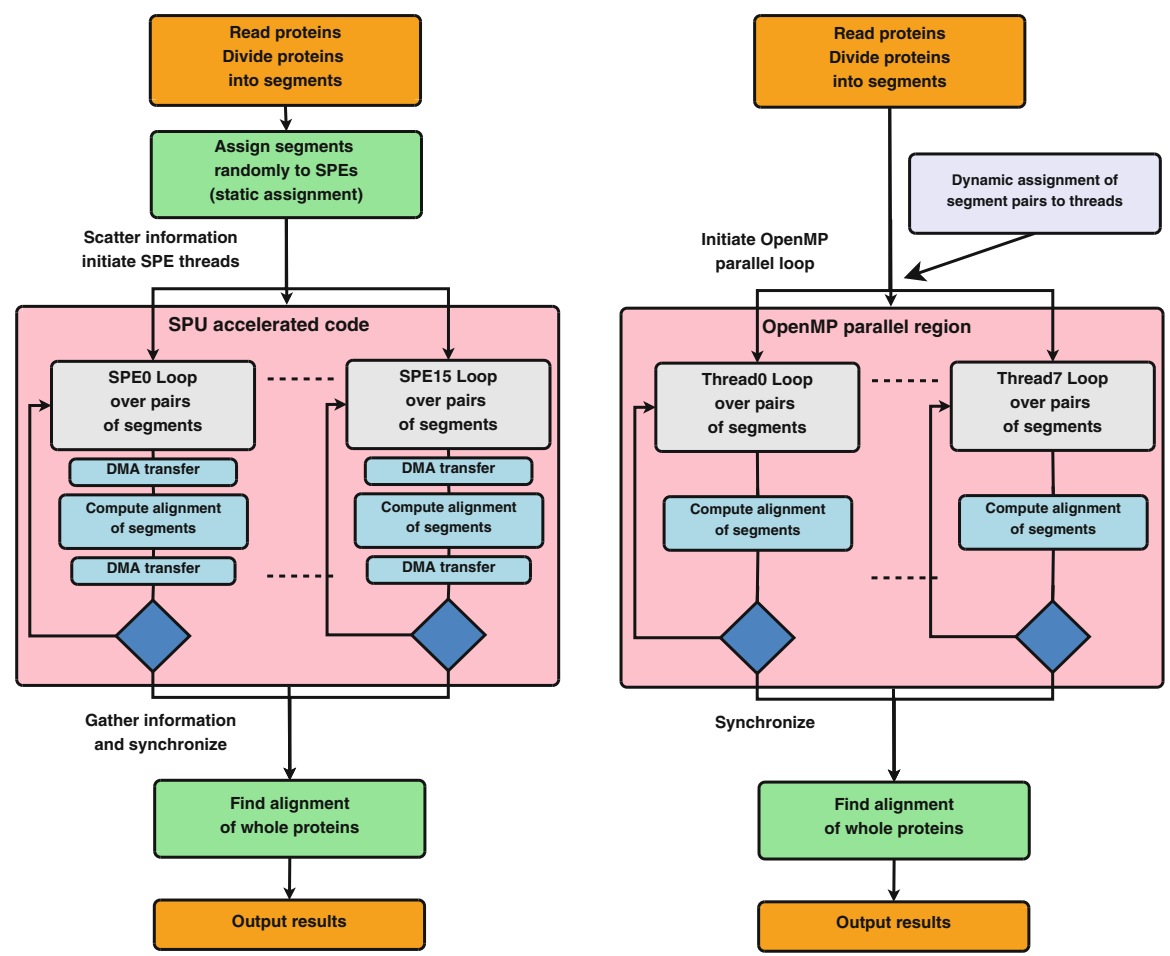

Fig. 2 The flow charts of the PowerXCell8i (left) and OpenMP (right) parallel versions of the 3D-Hit program. Important features of both implementations are indicated in the picture

$\frac{1}{(1-0.97994)+\frac{0.97994}{16}} \approx 12$. The difference between observed and expected values is the most probably due to the use of built-in permutation of packages instead of actually randomly permutated set of segments. This approach increases the probability of occurrence of inefficient load balancing.

\subsection{Performance Comparison}

We have designed a test to examine operational performance of 3 DHit code executing on various architectures. We have chosen a set of 18 proteins from a database and compared execution times on a Quad-Core AMD Opteron Processor 8354 based nodes and on a QS22 server. Each pair of proteins from the test set was compared during 
Table 5 Performance results on two systems: AMD Opteron 8354 and PowerXCell8i QS22

\begin{tabular}{llrl}
\hline Architecture & Compiler & Time (s) & Speedup \\
\hline AMD, 1 OpenMP thread & GCC (-03) & 116.85 & 1.00 \\
AMD, 4 OpenMP threads & GCC (-03) & 38.22 & 3.05 \\
AMD, 8 OpenMP threads & GCC (-03) & 26.72 & 4.37 \\
AMD, 16 OpenMP threads & GCC (-03) & 26.09 & 4.47 \\
PowerXCell8i, 1 SPE & GCC (-03 -ftree-vectorize) & 499.07 & 1.00 \\
PowerXCell8i, 8 SPEs & GCC (-03 -ftree-vectorize) & 81.20 & 6.14 \\
PowerXCell8i, 16 SPEs & GCC (-03 -ftree-vectorize) & 53.14 & 9.39 \\
\hline
\end{tabular}

a test run, which gave us 324 single test cases. The average results are presented in Table 5. As we can see, the Cell accelerated version of $3 D H i t$ was approximately two times slower than the OpenMP code executed on blades equipped with AMD processors. Those performance differences could be caused by disparities in technical parameters of chosen machines. According to our experiments, multiplication of two floating point scalars is almost two times slower on PowerXCell8i SPE processors than on AMD Opterons. Unfortunately a great deal of 3DHit code could not be vectorized and, as a result, arithmetic operations on scalars are intense in our code. Moreover, the PowerXCell8i version is slightly more complex. For example, the necessity of performing a memory optimisations as described above is a bottleneck on its own. Another very important feature that reduces the performance of the implemented SPU kernels is a big number of branching introduced by the algorithm and a lack of branch prediction mechanism within the SPU architecture. As a result one of the most important computational parts of the code, Smith-Waterman algorithm, is significantly slowed down on the Cell architecture.

On the other hand, the PowerXCell8i implementation has one desired feature, which is unfortunately not a feature of OpenMP-based program, namely-very good scalability. The reason of the bad scalability of OpenMP is probably due to the limited memory bandwidth when 16 working threads try to simultaneously read data from the shared memory located on their blade, which results in a significant slowdown of data transfer. It should be stated here that the comparison of two longest sequences in the benchmark test takes approximately only $0.22 \mathrm{~s}$. The memory bandwidth is not a problem in PowerXCell8i implementation, due to the presence of highly efficient Element Interconnect Bus (EIB), which provides each SPE and its memory controller with private and very fast connection to the main memory.

\section{Summary}

The current evaluation of 3 DHit performed on dataset of circa 300 query proteins reveals the quality of the tool, as compared with other programs. When compared with DALI server, our tool is able to generate similar number of correct models, however the final alignment quality is better in the case of the second service. In the case of distant structural comparisons, our method produced better ratings in all categories 
(such as specificity analysis) when MaxSub evaluation method is used. Concluding, the 3 DHit software is on average less sensitive than the DALI server, yet it is better than CE or VAST tools.

On the side of core optimization, we have ported the 3DHit program to the novel high-performance architectures and achieved very good rate of speedup. Our accelerated programs will be embedded into the web application, which will allow very fast and accurate mechanism for structural alignment of proteins. By taking advantage of PowerXCell8i and Quad-Core x86 novel architectures, we were able to significantly reduce time of single computation and as a result provide scientists all over the world with always up-to-date and fast tool for structural alignment experiments. The present version of our algorithm is very fast. It takes circa $2 \mathrm{~s}$ for a query protein of 500 amino acids to scan a database of 1,000 templates. The single comparison of two proteins with circa 500 amino acids is performed within a runtime of $0.002 \mathrm{~s}$ for 3 DHit, where older version of the software took around $0.017 \mathrm{~s}$. Other structural alignment programs are significantly slower, CE takes $3 \mathrm{~s}$ to compare two typical proteins, LGScore 2 is around $6 \mathrm{~s}$.

Because of its speed and portability (the source code is available from authors upon request) we believe that 3 DHit program will continue to be widely used in structural genomics projects, improving the structural comparison of newly crystallized proteins with large structural databases. We are planning to provide the internet web server interface to the PDB database, in order to give user access to rapid structural alignment of its protein of interest with three-dimensional crystals or 3D models of proteins.

Acknowledgments This work was supported by EC OxyGreen (KBBE-2007-212281) 6FP project as well as the Polish Ministry of Education and Science (N301 159735, and others).

Open Access This article is distributed under the terms of the Creative Commons Attribution License which permits any use, distribution, and reproduction in any medium, provided the original author(s) and the source are credited.

\section{References}

1. Nautilus system. http://cell.icm.edu.pl/index.php/Nautilus

2. The Green500 List. http://www.green500.org/lists/2009/06/list.php

3. Plewczynski, D., Pas, J., von Grotthuss, M., Rychlewski, L.: 3D-Hit: fast structural comparison of proteins. Appl. Bioinform. 1(4), 223-225 (2002)

4. Plewczynski, D., Pas, J., Von Grotthuss, M., Rychlewski, L.: Comparison of proteins based on segments structural similarity. Acta Biochim Pol. 51(1), 161-72 (2004)

5. Chothia, C., Lesk, A.M.: The relation between the divergence of sequence and structure in proteins. EMBO J. 5, 823-6 (1986)

6. Bujnicki, J.M.: Phylogeny of the restriction endonuclease-like superfamily inferred from comparison of protein structures. J. Mol. Evol. 50, 39-44 (2000)

7. Johnson, M.S., Sutcliffe, M.J., Blundell, T.L.: Molecular anatomy: Phyletic relationships derived from three-dimensional structures of proteins. J. Mol. Evol. 30, 43-59 (1990)

8. Berman, H.M., Westbrook, J., Feng, Z., Gilliland, G., Bhat, T.N., Weissig, H., Shindyalov, I.N., Bourne, P.E.: The Protein Data Bank. Nucleic Acids Res. 28, 235-42 (2000)

9. Arevalo, A., Matinata, R.M., Pandian, M., Peri, E., Ruby, K., Thomas, F., Almond, C.: Programming the PowerXCell 8i Architecture, Examples and Best Practices. RedBooks, IBM, USA (2008)

10. IBM \& ICM (University of Warsaw): Joint Cell Competence Center. http://cell.icm.edu.pl 
11. Plewczynski, D., Rychlewski, L., Jaroszewski, L., Ye, Y., Godzik, A.: SEA and FRAGlib—an integrated Web service for improving the alignment quality based on segments comparison. BMC Bioinform. 5(1), 98 (2004)

12. Holm, L., Kaariainen, S., Wilton, C., Plewczynski, D.: Using dali for structural comparison of proteins. Protoc. Bioinform. 5, 5.5.1-5.5.24 (2006) 